\title{
RNAi screening of the tyrosine kinome in primary patient samples of acute myeloid leukemia
}

\author{
David K. Edwards V1*, Fatma Eryildiz ${ }^{1 *}$, Jeffrey W. Tyner ${ }^{1}$ \\ ${ }^{1}$ Department of Cell, Developmental and Cancer Biology, Oregon Health \& Science \\ University, Portland, OR, USA \\ *these authors contributed equally to this work \\ Corresponding author: Jeffrey W. Tyner, tynerj@ohsu.edu
}

\begin{abstract}
Targeted therapy has proven to be successful in improving outcomes across multiple cancer types. However, many challenges still remain for implementation of this strategy in most patient cohorts, especially with the challenges of identifying the specific mutations or abnormalities in a heterogeneous tumor that are functionally significant. Previously, we developed a functional screening assay, RNAi-assisted protein target identification (RAPID) technology, which evaluates the viability of tumor cells after exposure to siRNAs against members of the tyrosine kinome and NRAS/KRAS. Here, we publish the comprehensive results of this screen for 332 primary AML patient samples. Data from these screening efforts have already helped identify previously unknown therapeutic targets, and will continue to provide insights into better treatment strategies for these patients.
\end{abstract}

\section{Introduction}

Acute myeloid leukemia (AML), a hematologic malignancy involving the proliferation of immature myeloid cells, is a heterogenous disease. ${ }^{1,2}$ Despite this intrinsic heterogeneity, targeted therapies have proven effective in AML subtypes with particular driver mutations. For example, for AML patients with FLT3 mutations, the kinase inhibitor, midostaurin, was shown to be more effective in combination with chemotherapy than chemotherapy alone. ${ }^{3}$ This finding resulted in midostaurin approval by the US Food and Drug Administration for use in newly diagnosed AML patients. There are numerous other efforts to implement this approach across different molecular subtypes, with varying degrees of success. ${ }^{4}$

One limitation to the implementation of this targeted therapy approach is in determining the molecular targets in AML that are functionally significant. To overcome this limitation, we have developed a method of screening primary AML patient cells, which we termed RNAi-assisted protein target identification (RAPID). ${ }^{5,6}$ Partial results from this screening technique have been published elsewhere ${ }^{7-14}$ although the entire dataset has never been released. 
Here, we are publishing the complete dataset containing the results of the RAPID screen performed on 332 primary AML patient samples. The significance of many of these kinases in the pathobiology of AML are currently being studied in our group and elsewhere.

\section{Methods}

Detailed methods for the RNAi-assisted protein target identification (RAPID) functional assay, including the electroporation conditions and the siRNA sequences, have been published elsewhere. ${ }^{5,6}$ Briefly, peripheral blood, bone marrow aspirates, or leukapheresis samples were obtained from AML patients by informed consent according to a protocol approved by the Oregon Health \& Science University (OHSU) Institutional Review Board. The peripheral blood mononuclear cells (PBMCs) were isolated from these samples using a Ficoll density gradient. These cells were added to a 96-well plate containing 91 arrayed siRNA pools that collectively target the tyrosine kinome, as well as siRNA pools targeting NRAS and KRAS. Following electroporation, the cells were transferred to basic cell culture media and incubated for 96 hours, at which time the cell viability was determined by a tetrazolium-based MTS assay.

We analyzed the data as reported previously. 5,6 Briefly, for each sample, we classified "hits", or siRNAs that had a significant impact on cell viability, as those whose viability was less than two standard deviations below the mean viability for all siRNAs on a given patient sample run. We performed unsupervised hierarchical clustering on the "hits" for each patient sample using Ward's method for clustering (the "hclust" function). The data was collected, analyzed, and visualized in $\mathrm{R}$.

\section{Results and Discussion}

In order to understand the results from our RNAi-assisted protein target identification (RAPID) functional assay, we graphed the frequency of "hits" that were observed in our 332 primary AML patient sample cohort (Figure 1).

The most significant "hit" in our screen was against CSF1R (colony stimulating factor 1 receptor), a class-III receptor tyrosine kinase that is expressed on monocytes and terminally differentiated macrophages. The large-scale sequencing effort of $200 \mathrm{de}$ novo AML patients undertaken by The Cancer Genome Atlas analysis did not identify any mutations in CSF1R, nor is it significantly overexpressed. ${ }^{1}$ (It should be noted that there are two cases of CSF1R mutations/translocations identified in AML cell linesspecifically, the Y571D mutation in GDM-1, which results in constitutively active CSF1R, ${ }^{15}$ and the $\mathrm{t}(3 ; 5)(\mathrm{p} 21 ; \mathrm{q} 33)$ RBM6-CSF1R translocation in MKPL-1, which renders it sensitive to CSF1R-targeting small-molecule inhibitors. ${ }^{16}$ However, these mutations have not been observed in any additional patient samples.) Our lab recently presented data to suggest that the significance of CSF1R in AML is related to the tumor microenvironment, ${ }^{17}$ and we are working further to uncover more information about the mechanism of action.

The second most significant "hit" was against JAK1 (Janus kinase 1), a nonreceptor tyrosine kinase involved with the activity of numerous cytokine signaling 
pathways. The significance of JAK1 mutations in AML is poorly understood. In a screen of the entire JAK1 coding region in AML patients, 2 out of 94 patients were found with JAK1 mutations. ${ }^{18}$ Although overexpression of these mutants did not transform Ba/F3 cells, they did show activation of downstream STAT1 and other signaling pathways. ${ }^{18}$ In addition, small-molecule inhibitors of JAK1 (and its related family member JAK2) are currently being investigated in relapsed or refractory AML patients. ${ }^{19,20}$ The role of JAK1 activation in the context of our patient sample cohort, however, is not fully understood.

The third most significant "hit" was against TYRO3 (TYRO3 protein tyrosine kinase), a member of the TAM family of receptor tyrosine kinases with a conserved extracellular structure and kinase domain sequence. Not much is known about the significance of TYRO3 in AML, although it has been shown to be upregulated in AML ${ }^{21}$ and targeting similar family members is an active area of investigation in leukemia. ${ }^{22} \mathrm{We}$ recently presented some data on the significance of TYRO3 in AML, ${ }^{23}$ although we are conducting more research to uncover its full significance.

Finally, the fourth most significant "hit", was against FLT3 (fms related tyrosine kinase 3), a class-III receptor tyrosine kinase whose involvement in AML has been wellstudied. FLT3 is one of the most commonly mutated proteins in AML, ${ }^{1,2}$ appearing either as an internal tandem duplication (FLT3-ITD) or a mutation in the tyrosine kinase domain (FLT3-TKD). This result is not unexpected, therefore, and serves as an indication that the results we observe in our patient sample screening conform with existing knowledge of AML pathobiology.

Finally, to identify any patterns of co-occurrence between siRNA hits among the patient samples, we performed unsupervised hierarchical clustering on the dataset (Figure 2). Overall, there were no significant clusters observed among the siRNAs, suggesting that each tyrosine kinase functioned independently of the activation of other tyrosine kinases. This result is not surprising, considering that there is a long-standing hypothesis that two classes of mutations are required to be present for leukemogenesis: disease-initiating mutations that block cell differentiation, and disease-progressing mutations that enhance cell proliferation and survival. ${ }^{24}$ Many of the diseaseprogressing mutations in AML occur in tyrosine kinases, specifically activating mutations in FLT3, KIT, and NRAS, ${ }^{25}$ so the activation of two kinase pathways could be functionally redundant for the development of leukemia. 


\section{References}

1. Cancer Genome Atlas Research Network. Genomic and epigenomic landscapes of adult de novo acute myeloid leukemia. N. Engl. J. Med. 2013;368(22):20592074.

2. Papaemmanuil E, Gerstung M, Bullinger L, et al. Genomic Classification and Prognosis in Acute Myeloid Leukemia. N. Engl. J. Med. 2016;374(23):2209-2221.

3. Stone RM, Mandrekar SJ, Sanford BL, et al. Midostaurin plus Chemotherapy for Acute Myeloid Leukemia with a FLT3 Mutation. N. Engl. J. Med. 2017;377(5):454-464.

4. Estey E, Levine RL, Löwenberg B. Current challenges in clinical development of "targeted therapies": the case of acute myeloid leukemia. Blood. 2015;125(16):2461-2466.

5. Tyner JW, Walters DK, Willis SG, et al. RNAi screening of the tyrosine kinome identifies therapeutic targets in acute myeloid leukemia. Blood. 2008;111(4):2238-2245.

6. Tyner JW, Deininger MW, Loriaux MM, et al. RNAi screen for rapid therapeutic target identification in leukemia patients. Proc. Natl. Acad. Sci. U.S.A. 2009;106(21):8695-8700.

7. Agarwal A, MacKenzie RJ, Eide CA, et al. Functional RNAi screen targeting cytokine and growth factor receptors reveals oncorequisite role for interleukin-2 gamma receptor in JAK3-mutation-positive leukemia. Oncogene. 2015;34(23):2991-2999.

8. Aslam MI, Abraham J, Mansoor A, et al. PDGFR $\beta$ reverses EphB4 signaling in alveolar rhabdomyosarcoma. Proc. Natl. Acad. Sci. U.S.A. 2014;111(17):63836388.

9. Bicocca VT, Chang BH, Masouleh BK, et al. Crosstalk between ROR1 and the Pre-B cell receptor promotes survival of $t(1 ; 19)$ acute lymphoblastic leukemia. Cancer Cell. 2012;22(5):656-667.

10. Carey A, Edwards DK V, Eide CA, et al. Identification of Interleukin-1 by Functional Screening as a Key Mediator of Cellular Expansion and Disease Progression in Acute Myeloid Leukemia. Cell Rep. 2017;18(13):3204-3218.

11. Javidi-Sharifi N, Traer E, Martinez J, et al. Crosstalk between KIT and FGFR3 Promotes Gastrointestinal Stromal Tumor Cell Growth and Drug Resistance. Cancer Res. 2015;75(5):880-891.

12. Maxson JE, Gotlib J, Pollyea DA, et al. Oncogenic CSF3R mutations in chronic neutrophilic leukemia and atypical CML. N. Engl. J. Med. 2013;368(19):17811790.

13. Sanda T, Tyner JW, Gutierrez A, et al. TYK2-STAT1-BCL2 pathway dependence in T-cell acute lymphoblastic leukemia. Cancer Discov. 2013;3(5):564-577.

14. Sun H, Lin D-C, Cao Q, et al. Identification of a Novel SYK/C-MYC/MALAT1 Signaling Pathway and Its Potential Therapeutic Value in Ewing Sarcoma. Clin. Cancer Res. 2017;23(15):4376-4387.

15. Chase A, Schultheis $B$, Kreil S, et al. Imatinib sensitivity as a consequence of a CSF1R-Y571D mutation and CSF1/CSF1R signaling abnormalities in the cell line GDM1. Leukemia. 2008;23(2):358-364. 
16. Gu T-L, Mercher T, Tyner JW, et al. A novel fusion of RBM6 to CSF1R in acute megakaryoblastic leukemia. Blood. 2007;110(1):323-333.

17. Edwards DK V, Rofelty A, Agarwal A, et al. CSF1R Inhibition Targets AML Cells By Depleting Supportive Microenvironmental Signal from CD14+ Monocytes. Blood. 2015;126(23):3824-3824.

18. Xiang $Z$, Zhao $Y$, Mitaksov V, et al. Identification of somatic JAK1 mutations in patients with acute myeloid leukemia. Blood. 2008;111(9):4809-4812.

19. Eghtedar A, Verstovsek S, Estrov Z, et al. Phase 2 study of the JAK kinase inhibitor ruxolitinib in patients with refractory leukemias, including postmyeloproliferative neoplasm acute myeloid leukemia. Blood. 2012;119(20):4614-4618.

20. Pemmaraju N, Kantarjian H, Kadia T, et al. A phase I/II study of the Janus kinase (JAK)1 and 2 inhibitor ruxolitinib in patients with relapsed or refractory acute myeloid leukemia. Clin Lymphoma Myeloma Leuk. 2015;15(3):171-176.

21. Linger RMA, Keating AK, Earp HS, Graham DK. TAM receptor tyrosine kinases: biologic functions, signaling, and potential therapeutic targeting in human cancer. Adv. Cancer Res. 2008;100:35-83.

22. Huey MG, Minson KA, Earp HS, DeRyckere D, Graham DK. Targeting the TAM Receptors in Leukemia. Cancers (Basel). 2016;8(11):101.

23. Eryildiz F, Tyner JW. Abstract 1265: Dysregulated tyrosine kinase Tyro3 signaling in acute myeloid leukemia. Cancer Res. 2016;76(14 Supplement):1265-1265.

24. Speck NA, Gilliland DG. Core-binding factors in haematopoiesis and leukaemia. Nat. Rev. Cancer. 2002;2(7):502-513.

25. Takahashi S. Current findings for recurring mutations in acute myeloid leukemia. $J$ Hematol Oncol. 2011;4(1):36. 


\section{Figure Legends}

Figure 1. Frequency of kinase "hits" in primary AML patient samples. Kinase targets and NRAS/KRAS screened using the RAPID assay are displayed on the $x$-axis and percentage of patients that showed significant sensitivity to the corresponding target siRNA exposure on the $y$-axis.

Figure 2. Heat map of kinase "hits" in primary AML patient samples. Patient specimen IDs are displayed on the $x$-axis and tyrosine kinases and NRAS/KRAS from the RAPID screening panel are displayed on the y-axis. siRNA "hits" are colored dark blue.

Supplemental Table 1. Complete dataset of RAPID screening results in primary AML patient samples. White blood cells from $332 \mathrm{AML}$ patients were processed and the sensitivity for each individual siRNA knockdown were measured as previously described. Data points below two standard deviations of the mean cell viability (at $P<$ 0.05 ) were considered "hits". Data shown here are the viability scores for each kinase from every plate per patient. 
bioRxiv preprint doi: https://doi.org/10.1101/256040; this version posted January 30, 2018. The copyright holder for this preprint (which was not certified by peer review) is the author/funder, who has granted bioRxiv a license to display the preprint in perpetuity. It is made available under aCC-BY-ND 4.0 International license.

Hit Ratio of Genes for Acute Myeloid Leukemia (AML) and Related Precursor Neoplasms

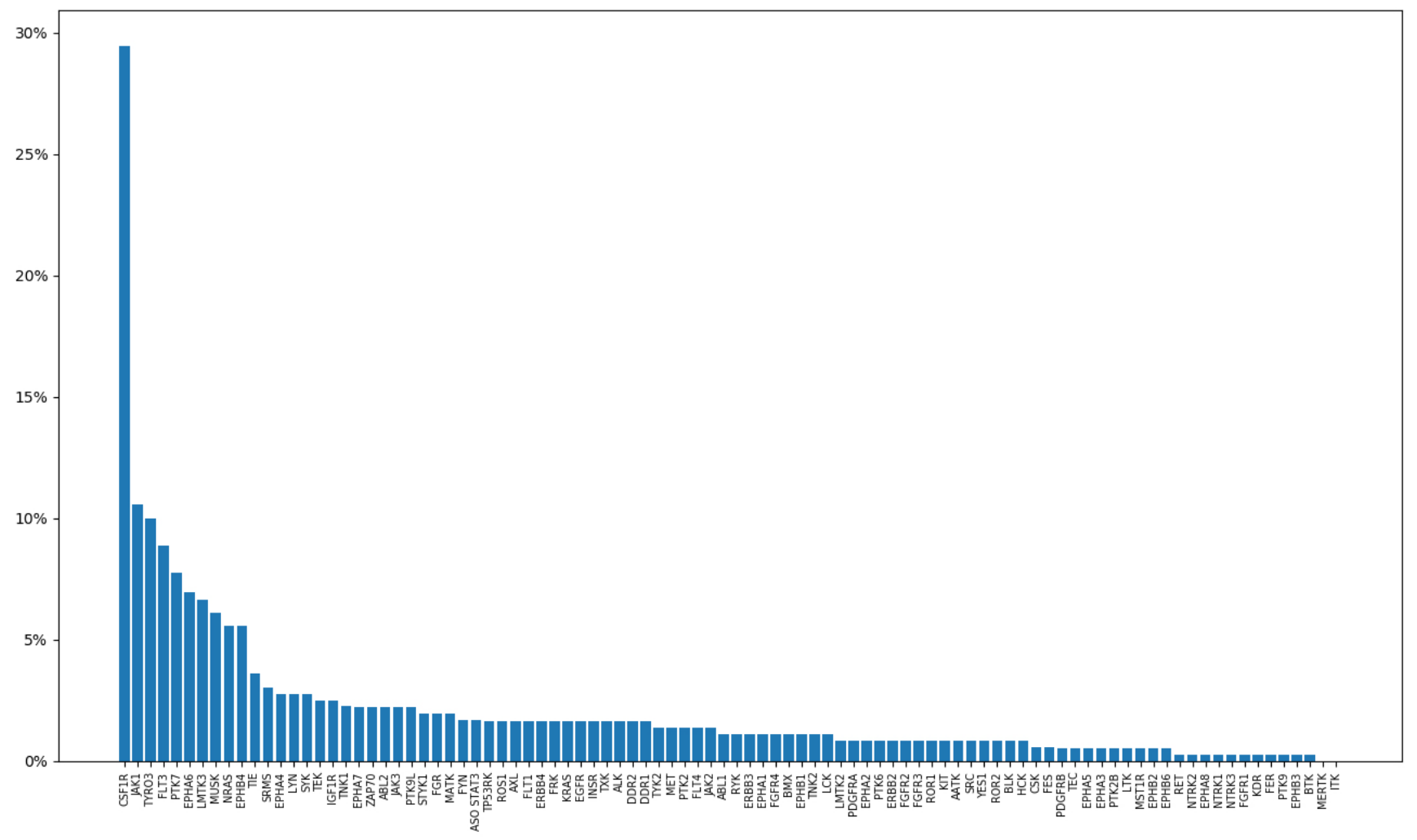


bioRxiv preprint doi: https://doi.org/10.1101/256040; this version posted January 30, 2018. The copyright holder for this preprint (which was not certified by peer review) is the author/funder, who has granted bioRxiv a license to display the preprint in perpetuity. It is made available under aCC-BY-ND 4.0 International license.

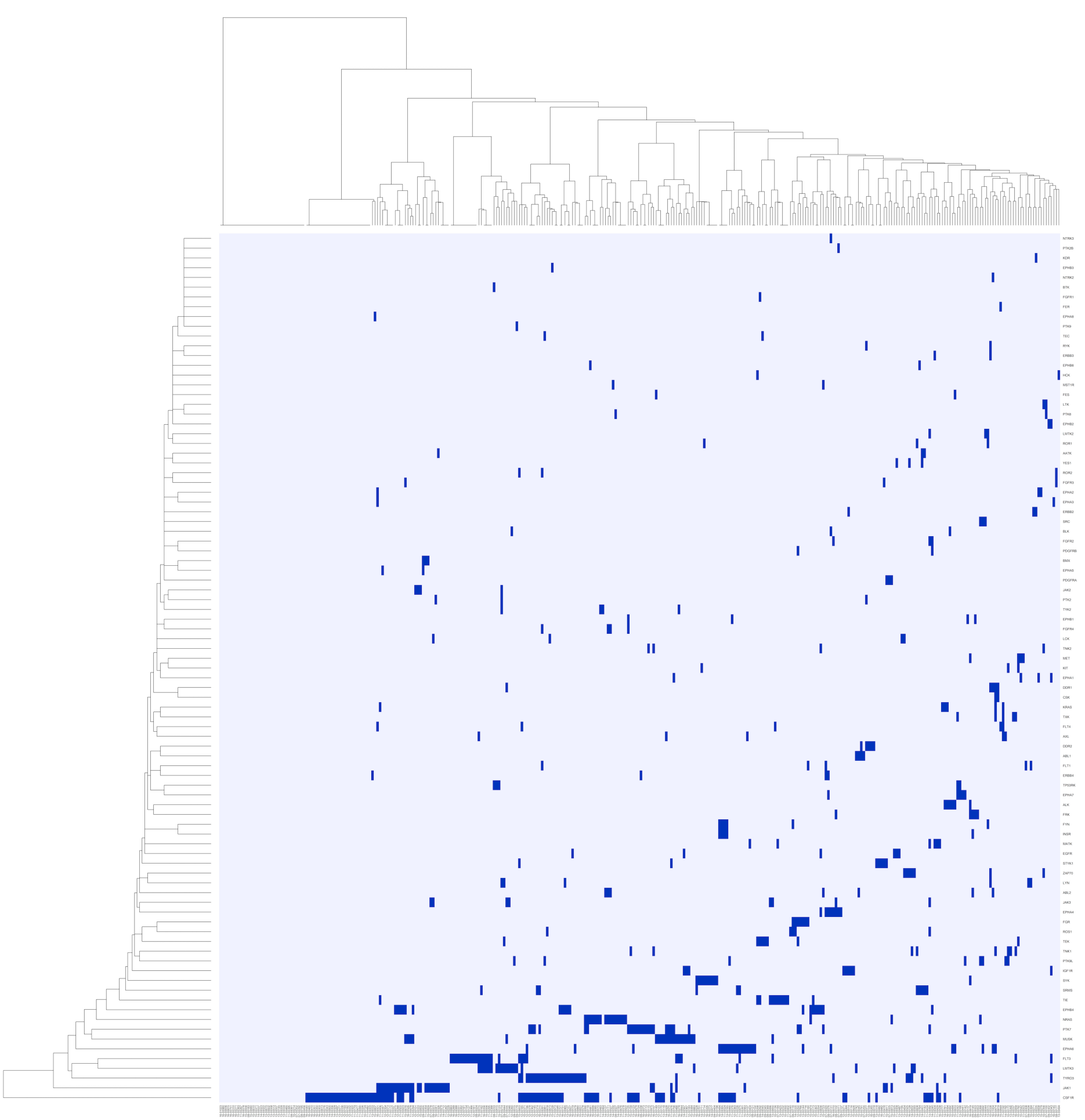

